\title{
Studiare dopo internet
}

\section{Alberto Sobrero, Università La Sapienza di Roma}

\begin{abstract}
The internet changes our ways of both knowing and thinking. A person who has grown up using the internet, whether for school or simply to play videogames, tends to extend and reproduce the same model of knowing in other instances. The internet replaces a linear and centralized model with a circular and diffused framework for self-learning that presents certain familiar limits: it demands speed or, at least, not pausing. The internet user is omnivorous, in a rush, and develops a memory that is limited to the short term. However, there are some positive aspects of circular learning that should be emphasized: the possibility of constructing personal paths, comparing different opinions, innovating, overcoming old disciplinary divisions, surmounting the drawbacks of linear learning that often lead to uncritical and purely molecular thinking.
\end{abstract}

La Rete modifica sia il modo di conoscere che di pensare. Una mente cresciuta sulla Rete, facendo ricerche per la scuola, o semplicemente giocando, tende a riprodurre anche in altre occasioni lo stesso modello di conoscenza. A un modello lineare la Rete sostituisce un modello circolare e diffuso di autoapprendimento che presenta alcuni noti limiti: richiede di correre, o per lo meno richiede di non fermarsi. L'utente della Rete è onnivoro e ha fretta e matura una memoria chiusa nel breve termine. Ci sono tuttavia aspetti positivi dell'apprendimento circolare che vanno sottolineati: la possibilità di costruirsi propri percorsi, sondare diverse opinioni, innovare, superare vecchie partizioni disciplinari, superando i difetti dell'apprendimento lineare che molto spesso induce a un pensiero acritico e premente molecolare.

Io mi colloco fra coloro che hanno incontrato abbastanza presto i problemi dell'informatica e coloro che sembrano rifiutare l'idea stessa che il mondo dei nostri studi stia radicalmente cambiando; fra quelli che riescono a tenere dietro alla rivoluzione permanente della Rete e quei colleghi che si disperano per l'ignoranza dei nostri studenti, e si meravigliano che anche i più bravi non abbiano letto Guerra e Pace, l'Ulisse, o Argonauti del Pacifico.

Eppure, diciamo trent'anni fa, mi avevano avvertito. Io ho cominciato a parlare di queste cose con tre maestri. E prima di fare alcune riflessioni generali li vorrei ricordare: sono Giorgio Cardona, Armando Petrucci e Alberto Cirese. In qualche modo tutti e tre anticiparono l'ingresso del computer nelle nostre stanze. 
Ognuno di loro, in tempi e situazioni diverse, mi ha insegnato qualcosa. Giorgio Cardona mi ha insegnato come il linguaggio del computer avrebbe cambiato non solo il modo di scrivere, ma principalmente il modo di pensare. Con Armando Petrucci ragionammo sulla possibilità e sulle difficoltà di un catalogo unico della letteratura popolare a stampa. La letteratura popolare non può definirsi se non nel suo contesto e questo è l'aspetto più difficile da formalizzare e che, dunque, rendeva Petrucci scettico sull'utilità del progetto.

Cirese il computer lo aveva atteso... lavorando con le schede perforate, con repertori, regesti, cataloghi. Nel 1979 a Villa Mirafiori entrò il Commodore con 32 K di RAM, poi il Commodore 64 e via via macchine sempre più potenti. Un po' tutti, con le lezioni di Carlo Cellucci, avevamo imparato a programmare in Basic. E il merito di Cirese fu quello di sottolineare subito la differenza tra un uso attivo e un uso passivo della macchina, la possibilità, come aveva scritto, di "comandare a un servo che non ha paura della morte" (Cirese). Fino agli ultimi anni Cirese continuò ad aggiornarsi, a studiare manuali. E penso che sia rimasto ingabbiato in questa contraddizione. Non si adattò mai all'idea che la macchina diventasse sempre più autonoma, sempre più senza padrone, o che il padrone fosse collettivo e invisibile. Con il computer voleva avere un rapporto personale, a due: un rapporto che finiva per essere inevitabilmente un rapporto con se stesso. Volle gestire il computer sfidandolo, come pensava fosse dovere di un intellettuale, ma forse finì per gestirlo con la mentalità di un intellettuale di altri tempi.

Queste premesse servono per introdurre alcune considerazioni generali sul tema assegnatomi: Studiare dopo internet. Il tema è vastissimo ed è difficile dire qualcosa di nuovo. Mi limito a offrire alcuni argomenti di discussione.

Comincio con il commentare due fra le affermazioni più frequenti intorno a questa questione. La prima è l'affermazione secondo la quale la Rete sta modificando il nostro modo di pensare e addirittura il nostro cervello. Per molti aspetti è un'affermazione vera, per altri meno. L'affermazione può essere assunta in tre versioni. Per capirci direi in una versione fortissima, in una versione forte e in una versione debole. Cominciamo non dalla prima, ma dalla versione forte, dall'idea che negli individui cambiamenti nella funzionalità e nello stesso assetto neurologico possano darsi in presenza di esperienze e sensazioni diverse. In questo senso penso che l'affermazione sia vera. L'assetto dei 100 miliardi (o quanti sono) di neuroni che abbiamo in testa è quanto mai plastico: e sia nella filogenesi che nell'ontogenesi questa è stata la proprietà più significativa del cervello. In antropologia cognitiva ci sono molte osservazioni su come le modalità della conoscenza possano modificare alcuni aspetti del complesso sistema di modulazione e demodulazione simbolica del mondo esterno e interno. E proprio Cardona aveva iniziato in Italia studi in questa direzione. Restando al nostro tema si potrebbe supporre, ad esempio, che lavorando con il computer per ore e ore si possa sviluppare di più l'emisfero sinistro rispetto al destro, le disposizioni analitiche rispetto a quelle di sintesi, l'elaborazione digitale rispetto alla percezione analogica. È vero, ad esempio, che alcune forme di memoria spazio-temporale tendono a potenziare le aree del cervello preposte all'orientamento spaziale. O molto semplicemente che l'esercizio della memoria potenzia i circuiti neuronali interessati al trasferimento delle informazioni nella memoria a lungo termine. Direi di più: come vedremo 
subito, stare molto al computer rende più difficile leggere libri; lo sperimentiamo ogni giorno e principalmente lo vediamo nei nostri studenti.

Ovviamente tutt'altra cosa è la versione fortissima della nostra affermazione, l'ipotesi che la macchina cerebrale possa, in relazione ai modi e ai contenuti della conoscenza, subire cambiamenti nella sua forma base, reintrodurre, insomma, un'ipotesi di tipo lamarckiano. Nel nostro caso, ad esempio, dovremmo dire che, nel lungo periodo, lo sviluppo del linguaggio digitale potrebbe diventare fattore selettivo nell'evoluzione della specie. E, dunque, che si vada verso la morte del libro per l'incapacità di leggerlo.

Non mi addentro in problemi evoluzionistici di questa natura. Lo scetticismo rispetto a un'interpretazione fortissima del rapporto fra macchina del cervello e modi del conoscere mi viene piuttosto da un altro aspetto della questione, dall'osservazione che, non dico il computer, ma la Rete è molto meno digitale di quanto normalmente si pensi. Una cosa è il metodo di manipolazione e trasmissione dei dati, la riduzione del mondo a un codice binario, e questo è il linguaggio del computer, altra cosa sono le disposizioni cognitive che dobbiamo mettere in atto per una ricerca in Rete, o davanti, ad esempio, a un testo multimediale.

Mettiamo da parte la vecchia immagine del cervello che conosce il mondo e sostituiamola piuttosto con la nozione di Mente, come fra i primi la intesero Maturana e Varela, e in campo antropologico Gregory Bateson, e come la intende ormai tanta scienza del Novecento: la Mente come insieme di sistemi che permettono a un organismo di scambiare con l'ambiente esterno informazioni, trasformando così se stesso e il proprio ambiente. Una pianta e il proprio ambiente formano una Mente, come qui dentro in questa giornata noi formiamo una Mente (almeno speriamo). Ogni uomo è il luogo di molte Menti, di molte relazioni, di molte storie. E allora si tratta di studiare i linguaggi della Mente, i sistemi di comunicazione attraverso i quali i messaggi vivono e si trasmettono all'interno della Mente, o meglio all'interno di quella che Bateson chiamava la Creatura, l'insieme delle Menti.

Non vorrei andare troppo avanti lungo questa pista e del resto sono argomenti ben noti, quel che mi interessa sottolineare è che non c'è una conoscenza analogica e una conoscenza digitale fra loro separate, ma che da sempre abbiamo comunicato con il mondo grazie a quel corpo calloso che lega fra loro i due emisferi e continueremo a pensare, a comunicare e a vivere in questo modo; questo è anzi uno dei caratteri precipui della nostra specie nel regno animale. I messaggi tra noi e il mondo, i messaggi attraverso i quali entriamo in relazione con il mondo e con noi stessi che siamo parte del mondo sono di varia natura, corrono lungo strade diverse, ma di certo sono sia analogici che digitali e probabilmente prevedono anche qualcosa di più, qualcosa su cui comunque qui non è il caso di fermarci.

Se la versione forte della nostra affermazione dice che la pratica di un pensiero digitale modifica l'assetto plastico del nostro cervello, la versione debole ci dice che questo accade da sempre, che da sempre la Mente ha avuto bisogno per comunicare con il mondo del linguaggio analogico e del linguaggio digitale, di un emisfero che ci rende parte del territorio e di un emisfero che dall'esterno ci costruisce mappe. 
Esclusa la versione fortissima, la versione forte può interessare i neuroscienziati: di certo è fondamentale vedere come la struttura plastica del cervello si conforma all'esperienza sensibile. Per noi antropologi interessante è piuttosto l'aspetto debole di questa affermazione, osservare come le modalità e i contenuti del conoscere siano strettamente connessi e come questo passi attraverso tutte le mediazioni e tutti gli strumenti che definiscono il rapporto fra noi e il mondo. Tra noi e il mondo c'è la storia della specie, ci sono gli altri, c’è la nostra cultura, ma anche la nostra capacità di manipolare il mondo, di costruire strumenti che potenziano la nostra presenza: dagli strumenti più semplici ai più raffinati. Dentro lo spazio della Mente c'è l'addomesticamento delle piante e degli animali, ma anche la stampa, il treno e ora il computer e la Rete. Con ognuna di queste innovazioni cambia in qualche modo la dimensione spazio-temporale della nostra Mente. Con molte differenze, ma in base ad analoghi principi.

Non ho bisogno di citazioni varie per dire che, a maggior ragione, l'insieme costituito da l'autore, dal testo e dal suo lettore è una Mente e che questa Mente è ovviamente diversa a seconda delle mediazioni che attraversa.

La Rete è una Mente e, come ogni altra Mente, è per sua natura dialogica, plurilogica. Che poi la sua configurazione la renda stupida o intelligente, è altra questione.

La seconda affermazione che vorrei brevemente commentare è quella secondo la quale dopo quella della scrittura, la rivoluzione della Rete, negli ultimi dieci-quindici anni, è la seconda vera rivoluzione culturale.

Anche in questa affermazione c’è indubbiamente molto di vero: la scoperta della stampa ha avuto una rilevanza sociale e culturale enorme e forse decisiva nel passaggio alla modernità, ma, in fondo, era un estremo perfezionamento della scrittura. E così per il computer nelle sue prime fasi. In ambedue i casi si trattava solo di uno sviluppo, per quanto immenso, delle capacità della memoria, un'estensione del deposito del sapere e della velocità della sua diffusione. Direi di più: c’è stato un breve periodo, pochi decenni, nei quali, rispetto alla scrittura, l'oralità sembrava destinata a recuperare il terreno perduto. La comunicazione sembrava scommettere più sulla radio, sui registratori, o sul telefono, che non sulla stampa o sulle fotocopie. Il passaggio alla rete ha segnato una svolta qualitativa: la Rete permette di sapere diversamente. E, a differenza di quanto è accaduto per la scrittura, nel caso della Rete la svolta è stata improvvisa. In questo senso non mi sembra esagerato usare il termine rivoluzione. Tutto vero, e sul quel sapere diversamente ci torneremo subito.

Ma, anche in questo caso credo che rimanga valido quel principio di Marshall McLuhan secondo cui “il ‘contenuto' di un medium è sempre un altro medium” (McLuhan 16). È un principio che può essere interpretato in molti modi, ma che proporrei di intendere non nel senso che il mezzo di comunicazione tecnologicamente più avanzato fagocita il precedente, né nel senso che lo sostituisce, ma semplicemente nel senso che sul piano della comunicazione i diversi sistemi di trasmissione ridefiniscono le loro postazioni e i loro campi di influenza, disegnano diversamente i loro confini: in molti casi si accostano semplicemente l'uno all'altro, in altri si escludono, si sovrappongono, o si fondono. 
Parliamo pure di rivoluzione, ma è bene sottolineare che anche in questo caso non c'è una linea evolutiva unica. Per dirla con l'immagine tradizionale non stiamo di fronte a un albero, ma a un cespuglio, a un moltiplicarsi e intrecciarsi di linee e possibilità di comunicazione. Quello che è ormai acclarato per l'evoluzione nel suo tratto biologico, vale anche nel tratto culturale. In questo senso proprio lo stesso studio della letteratura popolare insegna molto: come diceva una grande esperta della materia, la studiosa francese Geneviève Bollème, la letteratura popolare a stampa è una letteratura scritta da chi non sa scrivere per chi non sa leggere. E come nel caso della letteratura popolare bisogna intendere l'opposizione oralità versus scrittura in un senso molto debole, così si può osservare come nella Rete il rapporto fra i diversi sistemi di comunicazione sia quanto mai intrigato. Per certi versi è come se si fosse ingranata la marcia indietro. La stampa aveva superato i testi dei copisti, aveva escluso il rischio di errori, di falsificazioni, di correzioni, l'idea delle possibili varianti. I testi collaborativi della Rete ci riportano a prima della stampa, possono cambiare in continuazione, non sono mai definitivi. Ma questo è solo un primo passo, poi c'è il secondo; il ritorno, appunto verso l'oralità: come nei Forum e in Wikipedia.

Una cosa che consiglio sempre, a chi consulta Wikipedia, specie nei casi di voci apparentemente più neutre, è quella di cliccare sullo spazio Discussione. Ci sono voci corrette centinaia e migliaia di volte. Interventi che dissentono, che propongono, che rimandano ad altre voci, ad altri punti di vista. Alla formazione di Wikipedia collaborano, oggi 8 gennaio 2012, 703mila persone.

Si dice sempre del paradosso per il quale in Wikipedia gli argomenti più complessi sono quelli che faticano a trovare un equilibrio e quindi sono apparentemente trattati nel modo più neutro e quasi anonimo, ma bisogna appunto andare all'origine di quell'apparente anonimato, alla Discussione di cui la voce è il prodotto.

Vi inviterei, per esempio, a confrontare la voce bunga-bunga nell'edizione in inglese e in quella in italiano. In inglese:

Bunga bunga is a phrase of uncertain meaning that dates from 1910 if not earlier. By 2010 the phrase had gained popularity in Italy and the international press as well, when it was used by the Italian Prime Minister Silvio Berlusconi to refer to his alleged sex parties, which caused a major political scandal in Italy. ${ }^{1}$

In italiano, dopo lunga discussione e votata a maggioranza:

L'espressione XIX secolo, in particolare nella letteratura odeporica e nella manualistica botanica, prevalentemente come toponimo dell'Australia o come usanza aborigena della stessa nazione. Il termine è stato poi ripreso nel 1910, nell'ambito di un celebre scherzo, durante il quale ne è stata volutamente fatta intendere un'origine africana, con l'attribuzione di un significato incerto. ${ }^{2}$

È noto, del resto, come Nupedia, la prima Wikipedia, sia fallita proprio perché le voci venivano "controllate" da un team di esperti. Il passaggio da Nupedia a Wikipedia era il passaggio da una forma di scrittura pre-stampa, a qualcosa che assomiglia molto ai modi della comunicazione orale. 
La rivoluzione della Rete va intesa quindi non come un taglio netto, uno spartiacque fra il prima e il dopo, ma come un moltiplicarsi dei supporti e dei modi della comunicazione e un loro riassestarsi in confini ed equilibri diversi.

Facciamo esempi più seri. La voce Evoluzione. La voce inglese, prevede 279 references, un archivio di qualche centinaia di pagine di discussione, i pro e i contro, le prove, l'analisi dei testi, una catena infinita di rinvii etc. In questo caso anche la versione dell'edizione italiana (fatta se capisco bene da Telmo Pievani) ha suscitato dal 2005 un discreto dibattito, tecnico e ideologico. Ma prendiamo un'altra voce Cultura. Nel dizionario inglese, la voce prende varie pagine e la discussione dal 2004 a oggi è ininterrotta e archiviata anno per anno. Nella versione in italiano il testo è una versione ridotta della voce inglese, ma senza nessuna discussione.

Quel che spesso noi docenti non sappiamo fare e insegnare è non tanto saltare il baratro fra la comunicazione della lezione orale o del testo scritto e il nuovo flusso comunicativo della Rete, ma non vedere i ponti fra la vecchia e la nuova oralità, fra la vecchia e la nuova scrittura, all'interno della Rete. I molti possibili collegamenti e attraversamenti fra i diversi flussi di comunicazione. Molte cose sono cambiate, ma molte sono rimaste uguali e non potranno cambiare più di tanto, ma richiedono una gestione diversa.

È chiaro che, contrariamente a quanto di solito si pensa, l'interesse di Wikipedia sta spesso proprio nella molteplicità dei suoi linguaggi, nella pluralità delle fonti, nella non chiusura dei testi, più che nel testo finale. Più in quello che non appare che in quello che appare.

Noto solo un altro aspetto fra i tanti possibili. Se cercate, poniamo Lévi-Strauss in italiano, trovate un paio di pagine, delle quali, per esperienza, riconosco, o credo di riconoscere l'autore. La discussione è inesistente. Se andate nella pagina francese vi trovate ovviamente un articolo molto più significativo, ma principalmente se cliccate sullo spazio della discussione siete rinviati ai diversi progetti, in questo caso al projet d'anthropologie, dove Wikipedia nell'edizione francese è approfondita e adeguatamente francesizzata, come contenuti e come discussioni. Non è difficile vedere dietro l'impostazione dell'edizione francese di Wikipedia una politica culturale e una comunità scientifica che nell'edizione in italiano sembra del tutto assente.

Veniamo ora brevemente alla fruizione del mezzo da parte degli studenti. Wikipedia è nata se non sbaglio nel 2001 e le reti sociali - le varie MySpace, Facebook, etc. - si sono moltiplicate a partire dalla metà del passato decennio, e, dunque, gli studenti di oggi sono grosso modo la prima generazione nata culturalmente nella Rete.

Premetto che della crisi dell'università gli studenti sono davvero gli ultimi responsabili, né penso che la colpa si possa dare alla Rete. Sta di fatto che la Rete ha modificato tempi e modi dello studio e, in primo luogo, il rapporto con il libro. Sono cose che sappiamo bene, di cui si è discusso molto e di cui tutti abbiamo esperienza.

Una prima opposizione che spesso si sente ripetere è quella fra un apprendimento lineare e un apprendimento circolare. Il nostro tempo di studio era lineare, congeniale alla 
forma libro: come in un libro c'era un inizio e una fine, un sapere propedeutico, degli strumenti e un linguaggio di cui impossessarsi, e c'era un momento in cui si poteva dire di essere padroni di un certo argomento. Il percorso era per tutti uguale e confrontabile. E anche l'organizzazione della cultura e della società procedeva per via lineare: la divisione del lavoro fra chi scriveva un libro e chi lo leggeva, fra chi insegnava e chi apprendeva, i passaggi generazionali.

A un modello lineare la Rete sostituisce un modello circolare e diffuso di autoapprendimento, ma prima che nella Rete il tempo lineare viene meno nella società. Sono cose sulle quali c'è ormai una bibliografia vastissima. E sono noti i difetti del modello di apprendimento attraverso la Rete. L'apprendimento circolare richiede di correre, o per lo meno richiede di non fermarsi. L'utente della Rete è onnivoro e ha fretta; a ogni incrocio bisogna scegliere, ogni volta si scoprono possibilità e strade impreviste. Se provate a domandare a un vostro studente perché non ha letto questa o quell'opera di un certo peso, una delle risposte più frequenti è: "non ho tempo." La risposta suona bene, è quella famosa del matematico Galois: con la differenza che Galois il giorno dopo aveva un duello, mentre per lo più il nostro studente al massimo starà preparando un microesame.

Ma quella dello studente è una risposta sincera. Non c'è un inizio e non c'è una fine della ricerca; tutto è parte di un insieme senza confini. La ricerca potrebbe andare avanti all'infinito e dimenticare completamente il punto di partenza.

La Rete non dà la misura di quanto non si sa, come può capitare entrando in una biblioteca; è piuttosto una terra di mezzo fra il non sapere e il sapere. Basta andare in Rete! La psicologia della Rete è, del resto, un po' quella che accompagnava la nostra acquisizione di fotocopie: una volta fatte erano anche un po' studiate! Le conoscenze dei manuali (e magari i riassunti dei romanzi) sono tutte là, sempre disponibili e facilmente reperibili. La mediocrità, il carattere impersonale, la parcellizzazione, la fretta, sono caratteri connaturati alla logica della Rete.

Gli aspetti positivi sono l'altra faccia dei difetti. Da una parte il rischio di un pensiero acritico e molecolare, dall'altra la possibilità di costruirsi propri percorsi, sondare diverse opinioni, innovare, superare vecchie partizioni disciplinari, saperne più dei docenti.

Lo strumento della conoscenza, come dicevo, modifica non solo il modo di conoscere, ma anche il modo di pensare. Si pongono subito due problemi. Il primo riguarda la memoria. Tutti avrete notato come i nostri studenti dimentichino presto quel che hanno studiato. Il passaggio dalla memoria di lavoro, a quella a lungo termine dipende da due fattori: un adeguato tempo di esposizione allo stimolo e la capacità di elaborazione dello stesso, la possibilità di collocare il dato elaborato all'interno di un quadro di riferimento o, meglio all'interno di una storia già presente. La questione è molto più complessa: è interessante, ad esempio, notare che si memorizzano più facilmente i dati e le esperienze che si ritengono più utili, o che le esperienze e le notizie piacevoli o spiacevoli si memorizzano in maniera diversa. E questo accade anche per le nozioni, considerate più o meno interessanti. 
Anche limitandoci a una presentazione generale della questione, è chiaro che una lettura veloce e frammentaria come quella della pagina in Rete, ne rende difficile la memorizzazione. Tanto più che ogni pagina tende a essere indipendente e autosufficiente e, anzi, per lo più si tratta di frammenti troppo piccoli e troppo numerosi perché possano essere momentaneamente depositati nella memoria di lavoro ed essere elaborati, considerando, come è noto, che la memoria a breve termine più di tanto non può contenere.

Gli esperimenti in questo senso sono numerosi e tutti arrivano alla stessa conclusione. Durante la lettura di una pagina scritta si registra un'attività cerebrale decisamente superiore a quella registrata durante la lettura di pagine in Rete, si ricorda molto di più quando si legge da un libro che quando si legge nella Rete, e mentre nel primo caso si è in grado di ricostruire il contenuto della lettura nell'ordine in cui è esposto, nel secondo caso il ricordo oltre ad essere decisamente inferiore è anche del tutto disorganizzato. Si tende anzi, a ricordare come quando si ricordano i sogni: ognuno ricorda a proprio modo, ma tutti ricordiamo a partire dall'ultima pagina per tentare di risalire alla prima.

Non posso approfondire la questione, ma è chiaro che siamo in presenza di due memorie completamente diverse, e una mente cresciuta sulla Rete, facendo ricerche per la scuola, o semplicemente giocando, tende a riprodurre anche in altre occasioni lo stesso modello di conoscenza. Leggere un libro diventa faticoso, specie se si tratta di un romanzo, di una storia lunga e che non può essere frammentata più di tanto. I romanzi pretendono una lettura profonda e sembrano incompatibili con la Rete.

Ma c’è un ultimo aspetto della questione che vorrei almeno sfiorare, è l'interrogativo che si è posto Aime in un recente libretto, Il dono al tempo di Internet. Come sapete si tratta di un argomento molto trattato in antropologia: qual è il significato di un dono? Perché si dona? Da Mauss in poi il dibattito è stato intenso. Quali sono le motivazioni che spingono a contribuire allo scambio di file, di conoscenze, di competenze culturali? Quali sono le motivazioni che portano a condividere, a mettere in Rete gli appunti di una lezione, o a rispondere alla richiesta di aiuto sul passaggio di un testo, sulla miglior traduzione di una certa frase idiomatica, o semplicemente sull'uso di una parola?

In Italia non abbiamo ancora in ambito antropologico una riflessione sul rapporto fra antropologia e informatica analoga a quella che hanno i filologi, o progetti complessivi come quelli degli storici (Bologna). In Italia pochissimi antropologi, docenti o studenti che siano, collaborano, per esempio, a Wikipedia. Molto ridotta è anche la produzione di software specifici, quasi esclusivamente dedicati alla catalogazione del patrimonio culturale (BravoTucci, Costa). In linea di massima i siti e i portali di cattedre, dottorati, musei, riviste, come Antropologie (Clemente), Fareantropologia (Dei) o come Anthropolis, sono pagine non molto ricche.

E solo da poco si è assunto il mondo della Rete come possibile terreno di ricerca etnografica e di riflessione antropologica (Bitti, Dei).

Il limite di queste riflessioni mi sembra quello di utilizzare troppo genericamente il termine Rete, o termini come File sharing etc. Bisognerebbe a mio avviso fare alcune distinzioni. Anche se accettiamo una definizione di dono molto più vicina a quella di 
Derrida che non a quella di Mauss e quindi diciamo che lo scambio in Rete è uno scambio generalizzato e allargato, un dono fatto anonimamente a una comunità anonima, senza pensare di poterne avere una qualche restituzione immediata e senza contabilizzare il dare e il ricevere, dobbiamo, tuttavia, fare alcune distinzioni in base ai contenuti scambiati e alle comunità coinvolte. Distinguerei decisamente fra comunità senza confine, come nel caso di Wikipedia, e comunità definite, o per interessi, o in riferimento a confini esterni. La differenza è notevole.

Diverso è il caso dei Forum free studenteschi. Prendo ad esempio il Forum degli studenti del Corso di Laurea di antropologia di Lettere alla "Sapienza" di Roma. Quasi tutti gli studenti dichiarano di entrare nel Forum, di prendere sul serio le indicazioni che vi trovano, di collaborare con loro opinioni. E questo penso capiti un po' per tutti i corsi di laurea. È un materiale che meriterebbe una qualche attenzione. Perché uno studente perde tempo (fra virgolette) a spiegare a un altro studente i sistemi fondamentali della parentela o la nozione di rappresentazione perspicua in Wittgenstein? Penso ad alcune risposte possibili.

Di certo, in primo luogo, per un bisogno di appartenenza, per il bisogno di affermare la propria presenza all'interno di un gruppo. Di solito gli interventi sono seri, rispettano i principi della netiquette, raramente trovo meccanismi espliciti di competizione. Ed è un bisogno di presenza tanto più grande quanto più l'istituzione universitaria è lontana e silenziosa. Non ci si aspetta alcun ritorno, e non mi sembra ci sia una vera circolarità. A rispondere sono sempre un gruppo di nickname evidentemente più preparati, per i quali segnare la propria presenza competente confina in molti casi con la necessità di conferma, di autostima, e magari con un evidente e salutare narcisismo.

C’è poi una seconda motivazione che mi sembra decisiva. Preso nel suo complesso questo genere di scambio configura non tanto un'opposizione tra logica del mercato e logica del dono, come nel caso di Wikipedia o di altre forme di condivisione p2p, quanto un'opposizione alla logica dell'istituzione in quanto tale, una sorta di alleanza tattica contro il sistema. Il linguaggio, per quanto rispettoso, rivela una guerriglia semantica più vicina alle tattiche subalterne di resistenza studiate da De Certeau che non a un progetto politico generale di contestazione.

In qualche modo difetti e pregi della Rete si riflettono nella psicologia della nuova generazione (o ne sono riflesso). Sono i caratteri dei recenti movimenti studenteschi: l'assenza di grandi ideologie, l'assenza di progetti alternativi, un procedere a volte confuso, ma, al tempo stesso, la capacità di non farsi intrappolare in schemi e partiti, di inventare percorsi nuovi, l’idea di prendere possesso della città dirigendosi improvvisamente verso la periferia piuttosto che marciare verso il centro.

Quel che voglio dire è molto semplice: dobbiamo ripensare le forme del nostro linguaggio, più di quanto non si sia fatto fino adesso, e fare pendere la bilancia dalla parte degli aspetti positivi della Rete. Non è detto, ma è solo un esempio, che un romanzo di cento o cinquecento pagine vada letto solo in modo lineare: si può insegnare a smontarlo e rimontarlo, a identificare percorsi diversi, a scoprirne le parentele in altri romanzi, a 
ipotizzare altri finali. Non è questa del resto, la vera natura del romanzo? Quello che chiedevano di fare tanti romanzieri, da Cervantes e Diderot in poi? E così la lettura di un saggio può attraversare molti altri saggi, girare nella Rete, arricchirsi, riempirsi di ipotesi alternative, sperimentare nuove forme di rappresentazione. E questo è forse più importante nelle nostre discipline umanistiche che non nelle discipline scientifiche.

Se vediamo così le cose, i problemi non sono quelli che ci tormentano da dieci anni: il rapporto fra crediti e numero di pagine, le richieste di programmi in "non più di cinque righe, con traduzione in inglese, dei risultati attesi," o stupidaggini simili. Si può portare all'esame anche un solo romanzo o un solo saggio, ma lo si può far esplodere nelle più impensate direzioni. Come accade nella Rete, le cento pagine possono diventare mille. Si tratta, per così dire, di marcare percorsi lineari all'interno di sistemi complessi, fino a imparare a leggere l'intero romanzo proustiano, o fino a studiare Le strutture elementari della parentela, insegnando il piacere di quella lettura, leggendo ogni pagina come un passaggio inatteso.

${ }^{1}$ http://en.wikipedia.org/wiki/Bunga bunga [9 gennaio 2012].

${ }^{2}$ http://it.wikipedia.org/wiki/Bunga bunga [9 gennaio 2012]. 


\section{Works Cited}

Aime, Marco e Cossetta, Anna. Il dono al tempo di Internet. Torino: Einaudi, 2010. Print.

Bateson, Gregory. Mente e Natura. Un’unità necessaria. Milano: Adelphi, 1984. Print.

Bollème, Geneviève, ed. La Bibliothéque Bleu. La littérature populaire en France du XVIIe au XIXe siècle. Paris: Gallimard/Julliard, 1971. Print.

Bollème, Geneviève. Les almanachs populaires aux 17. et 18. siècles. Essai d'histoire sociale. Paris: Mouton \& Co., 1969. Print.

Cirese, Alberto Mario. "Il potere del computer: come comandare a un servo che non ha paura della morte.” Potere senza stato. Atti del convegno. Cagliari 10-11 maggio 1984. Ed. Carla Pasquinelli. Roma: Editori Riuniti, 1986. 163-181. Print.

de Certeau, Michel. L'invenzione del quotidiano. Roma: Edizioni Lavoro, 2001. Print.

Derrida, Jacques. Donner le temps, Paris: Galilée, 1991; Donare il tempo. La moneta falsa. Milano: Raffaello Cortina, 1996. Print.

Mauss, M., 1924, "Essai sur le don. Forme et raison de l'échange dans les sociétés archaïques." L’Année Sociologique, 1, pp. 30-186; Saggio sul dono. Forma e motivo dello scambio nelle società arcaiche. Torino: Einaudi, 2002. Print.

Maturana, Humberto; Varela, Francisco. Autopoiesi e cognizione. La realizzazione del vivente. Venezia: Marsilio, 1985. Print.

McLuhan, Marshall. Gli strumenti del comunicare. Milano: Net, 2002. Print.

Wikipedia. "Bunga bunga” Wikipedia, L'enciclopedia libera, 2011-2012. Web. 9 gennaio 2012. 